Methods We describe a 5-year-old boy who suffered from chronic recurrent severe lip angioedema for more than 2-years. His symptom was not associated with facial rash or any food ingestion. He was also suffering from dental carries therefore dental extraction was performed for that possibly of secondary gingivitis associated lip angioedema, but without improvement. In occasions he had oral ulcers and the possibility of Behçet's disease was considered for which colchicines was used for more than 3-weeks without benefits. Cervical lymph node biopsy ruled out malignancies.

Results He was found to have chronic congested nasal mucosa. Skin prick test (SPT) for environmental allergens was strongly positive for hose dust mites, cockroaches, grass and molds. Nasal findings and SPT confirmed the diagnosis of AR. Over 2-months he has significant improvement (> 70\%) in his symptoms with AR treatment (ie; fluticasone spry BID, montelukaste $4 \mathrm{mg}$ PO OD and loratidine $2.5 \mathrm{mg}$ PO OD). Normal C1 esterase level and function ruled out hereditary angioedema.

Conclusion AR is a common disease that should be considered in the differential diagnosis of chronic recurrent lip angioedema. Diagnosis in children may be delayed because of the overlapping diseases (i.e.; viral upper respiratory tract infections). However, hereditary angioedema needs to be ruled out as an important cause of recurrent angioedema.

\section{POST-BRONCHIOLITIS RECURRENT WHEEZING IN CHILDREN UNDER 2 YEARS OF AGE - A CLINICAL EPIDEMIOLOGICAL STUDY}

doi:10.1136/archdischild-2012-302724.0478

'SC Cosoveanu, 'IO Petrescu, ${ }^{2} \mathrm{M}$ Ganea. ${ }^{12 n d}$ Pediatric Clinic, University of Medicine and Pharmacy Craiova; ${ }^{2} 2$ nd Pediatric Clinic, Emergency County Hospital Craiova, Craiova, Romania

Objectives The research involved tracing some causality relationships among acute bronchiolitis, post-bronchiolitis recurrent wheezing and/or the atopic field.

Material and method The study group was made up of 118 cases of acute bronchiolitis in the children aged 0-2 years, admitted to the $2^{\text {nd }}$ Pediatric Clinic in Craiova, in 2011. Traceable parameters: demographic (sex ratio, age, gestational age, birth weight, socio-economic environment), risk factors (history of pulmonary/heart disease, previous to bronchiolitis onset, prematurity), personal or/and family history of atopy, nutritional state, clinical and radiologic aspects, evolution under treatment, period of hospitalization.

Results Acute bronchiolitis prevalence was of $5 \%$. Most cases were registered in the 6 to 12 months age group (43.2\%), males (69.5\%), in rural areas (56\%), and during cold, wet season (70.2\%). $63.6 \%$ of the cases had low birth weight. Dystrophy was present in $15.2 \%$, carential rickets $46.7 \%$, carential anemia $63.5 \%$ of the cases. $28 \%$ of the cases developed recurrent episodes of wheezing associated with lower respiratory tract infections. Radiologic images of pulmonary condensation were present in $77 \%$ cases with severe bronchiolitis. Severe bronchiolitis form was associated to a hospitalization period of 7-14 days. The atopic aspect was noticed in $9.3 \%$ of the cases. Children with atopy history had a longer period of symptoms and more days of hospitalization as compared to the ones without atopy antecedents.

Conclusions Cigarette smoke exposure, carential rickets, dystrophy, low birth weight, atopy association, all represented risk factors which significantly contributed to the appearance of postbronchiolitis recurrent wheezing in infants and young children.

\section{BIOLOGICAL DIAGNOSIS OF BORDETELLA INFECTIONS AND DETERMINATION OF THEIR EPIDEMIOLOGICAL CHARACTERISTICS IN TUNISIAN INFANTS $<1$ YEAR OF AGE}

doi:10.1136/archdischild-2012-302724.0479
'A Zouari, ${ }^{1} \mathrm{H}$ Smaoui, ${ }^{2,3} \mathrm{D}$ Brun, ${ }^{2,3} \mathrm{E}$ Njamkepo, ${ }^{4} \mathrm{E}$ Zouari, ${ }^{5} \mathrm{~K}$ Menif, ${ }^{5} \mathrm{~N}$ Ben Jaballah, ${ }^{2,3} \mathrm{~N}$ Guiso, 'A Kechrid. 'Biology, Laboratory of Microbiology, Children's Hospital of Tunis, Tunis, Tunisia; ' $M o l e c u l a r$ Prevention and Therapy of Human Diseases, Pasteur Institute, ${ }^{3}$ URA CNRS 3012, Paris, France; ${ }^{4}$ Marketing, Business School IHEC; ${ }^{5}$ Paediatric Intensive Care Unit, Children's Hospital of Tunis, Tunis, Tunisia

Background and Aims Bordetella pertussis and B. parapertussis are the causative agents of whooping cough, a re-emerging infectious disease in spite of reasonable vaccination coverage. Specific diagnostic tools were applied for the first time in a Tunisian prospective study in order to get an estimation of the prevalence of Bordetella infections, and to evaluate their use to determine the epidemiological characteristics of these infections in Tunisian infants.

Methods Between 2007 and 2011, a total of 626 samples from 599 infants aged $<1$ year with and without pertussoid cough were investigated for the presence of $B$. pertussis/parapertussis using culture and real-time PCR (RT-PCR). When possible, patients' household contacts provided nasopharyngeal aspirates (NPAs) for RT-PCR detection of $B$. pertussis/parapertussis or single-serum samples for anti-PT IgG quantification.

Results All except 1 NPA were negative by conventional culture whereas PCR gave positive signals for 126 specimens (21\%): B. pertussis, B. parapertussis and Bordetella spp. were detected in 82, 6 and $4 \%$ of the samples, respectively. The simultaneous presence of B. pertussis and B. parapertussis was noted in $8 \%$ of the cases. The prevalence of Bordetella infection was $20 \%$. Most of these cases corresponded to patients younger than 6 months old who received $<3$ doses of pertussis vaccine. Among the household contacts enrolled in the study, mothers were the likely source of infection in 4 cases. Conclusions This study showed that pertussis is still prevalent in Tunisia and the disease remains a public health problem affecting not only infants but also adults.

\section{SUCCESSFUL BLO0D SAMPLE SALVAGING FROM PRETERM INFANTS: MAXIMISING RESEARCH OPPORTUNITIES, MINIMISING PARENTAL AND INFANT INTERVENTIONS}

doi:10.1136/archdischild-2012-302724.0480

1.2J Berrington, ${ }^{2} \mathrm{E}$ Turnbull, ${ }^{3} \mathrm{~S}$ Turner, ${ }^{3} \mathrm{~S}$ Douglas, ${ }^{1,2} \mathrm{~N}$ Embleton. ${ }^{1} / \mathrm{nstitute}$ of Health and Society, Newcastle University: ${ }^{2}$ Neonatal Medicine: ${ }^{3}$ Biochemistry, Newcastle upon Tyne Hospitals NHS Foundation Trust, Newcastle upon Tyne, UK

Backround and aims Research improves care for preterm infants but taking blood may be inappropriate/ethically challenging, and limited by patient size. We aimed to 'salvage' residual blood after routine testing, and sought parental perspectives.

Methods We gave parents a single information leaflet and asked permission to collect non-invasive or 'un-used' samples to support a portfolio of research studies: stool/breast milk (gut microbiome), serum (cytokine), and DNA from residual cell pellet (epigenetic) We also sought consent for sample sharing with other approved studies. Parents could decline specific aspects. Serum/cell salvage was stored prior to analysis.

Results $99 \%$ of parents participated: 92 recruits provided 527 salvaged blood samples supporting two additional studies to date.

\section{Abstract 480 Table 1}

\begin{tabular}{ll}
\hline & Infants (parents) \\
\hline Total approached & $92(81)$ \\
Total agreeing to any aspect & $91(80)$ \\
Total agreeing to all aspects & $82(76)$ \\
Total declining all aspects & $1(1)$ \\
Total declining specific elements (of which:) & $9(6)$ \\
declining any salvaged blood & $1(1)$ \\
declining salvaged DNA & $6(4)$ \\
Declining specimen sharing & $2(1)$ \\
\hline
\end{tabular}

